\section{Statistical analysis of simultaneous monostatic and bistatic sea clutter at low grazing angles}

W.A. Al-Ashwal, C.J. Baker, A. Balleri, H.D. Griffiths, R. Harmanny, M. Inggs, W.J. Miceli, M. Ritchie, J.S. Sandenbergh, A. Stove, R.J.A. Tough, K.D. Ward, S. Watts and K. Woodbridge

Presented is a statistical analysis of vertically-polarised bistatic sea clutter data, with bistatic angles ranging from $30^{\circ}$ to $90^{\circ}$, compared with simultaneously-gathered monostatic clutter data. It is found that there is no significant change in the bistatic reflectivity compared to the monostatic except close to bistatic angles of $90^{\circ}$. It is found that the shape parameter of the K-distribution fitted to the clutter is significantly higher for the bistatic clutter than for the equivalent monostatic clutter.

Introduction: Despite significant current interest in bistatic radar, bistatic clutter is poorly understood [1]. There have been very few published results from bistatic sea clutter trials, and of these even fewer include the out-of-plane geometry $[1,2]$.

Good models of sea clutter are needed throughout the design cycle of a radar [3]. Significant advances have been made in the characterisation and modelling of monostatic sea clutter [4]; similar understanding of bistatic sea clutter is needed to be able to properly design and optimise bistatic radars.

Description of trials: NetRAD is a three node multistatic netted radar system [5, 6] developed at University College London (UCL). The current system uses GPS disciplined oscillators (GPSDOs) developed at the University of Cape Town (UCT) at each node to provide carrier and trigger synchronisation without the need for interconnecting cables [7]. The principal system parameters are shown in Table 1.

\section{Table 1: NetRAD parameters}

\begin{tabular}{|c|c|c|}
\hline Parameter & Monostatic node & Bistatic node \\
\hline Carrier frequency & $2.4 \mathrm{GHz}$ & $2.4 \mathrm{GHz}$ \\
\hline Nominal bandwidth & $50 \mathrm{MHz}$ & $50 \mathrm{MHz}$ \\
\hline Beamwidth & $9^{\circ}(\mathrm{az}) \times 11^{\circ}(\mathrm{el})$ & $9^{\circ}(\mathrm{az}) \times 11^{\circ}(\mathrm{el})$ \\
\hline Height above sea level & $12 \mathrm{~m}$ & $10 \mathrm{~m}$ \\
\hline Rx losses and noise figure & $7.7 \mathrm{~dB}$ & $5.4 \mathrm{~dB}$ \\
\hline Tx losses & $2 \mathrm{~dB}$ & - \\
\hline Transmitted power & $500 \mathrm{~W}$ & - \\
\hline PRF & $1 \mathrm{kHz}$ & - \\
\hline
\end{tabular}

In October 2010 UCL and UCT conducted monostatic and bistatic sea clutter and small maritime target signature measurements in the Western Cape of South Africa, using two of the three radar nodes. The measurements were carried out using vertical polarisation. The data analysed here is a subset of the data recorded at Cape Point on 5 October 2010. The bistatic geometry is shown in Fig. 1.

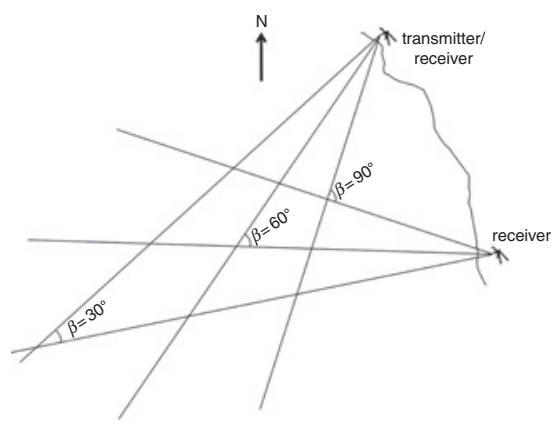

Fig. 1 Geometry for bistatic measurements

Baseline separation between nodes was $416 \mathrm{~m}$; orientation of baseline was 330 with respect to True North

Monostatic ranges corresponding to each bistatic angle listed in first column of Table 2
Table 2: Summary of results

\begin{tabular}{|c|c|c|c|c|c|c|c|}
\hline$R M(\mathrm{~m})$ & $\beta$ & $\begin{array}{c}\sigma_{M}^{0} \\
\left(\mathrm{~dB} \mathrm{~m}^{2} / \mathrm{m}^{2}\right)\end{array}$ & $\begin{array}{c}\sigma_{B}^{0} \\
\left(\mathrm{~dB} \mathrm{~m}^{2} / \mathrm{m}^{2}\right)\end{array}$ & $\begin{array}{c}\mathrm{CNR}_{M} \\
(\mathrm{~dB})\end{array}$ & $\begin{array}{c}\mathrm{CNR}_{B} \\
(\mathrm{~dB})\end{array}$ & $v_{\mathrm{M}}$ & $v_{\mathrm{B}}$ \\
\hline 805 & $30^{\circ}$ & -59.2 & -58.7 & 18.1 & 21.5 & 0.93 & 3.11 \\
\hline 417 & $60^{\circ}$ & -47.1 & -47.6 & 36.9 & 40.2 & 0.22 & 0.48 \\
\hline 295 & $90^{\circ}$ & -44.1 & -55.8 & 41.1 & 35.6 & 0.17 & 1.04 \\
\hline
\end{tabular}

Parameters are defined in text; subscripts $M$ and $B$ refer to monostatic and bistatic, respectively.

The transmitter and receiver locations were measured using the GPS in the GPSDO of each node. The bistatic baseline was approximately $416 \mathrm{~m}$. The bistatic angle $\beta$ was varied by rotating the antennas in azimuth, thus changing the intersection point of the beams. In all cases the bistatic triangle was isosceles, as shown in Fig. 1. The local grazing angles varied from approximately $2.3^{\circ}$ at the closest range to $0.8^{\circ}$ at the farthest range.

Simultaneous meteorological data gave the significant wave height as $1.3 \mathrm{~m}$ with swell propagating towards the shore, approximately sea state 2 . The wind speed was approximately $4 \mathrm{~ms}^{-1}$ from a southerly direction.

Average reflectivity: To estimate the normalised backscatter clutter coefficient from bistatic radar data, it is necessary to account for the changes of the range to the transmitter and receiver over the illuminated area, which depends both on the intersection of the antenna beams and on the pulselength, and is a truncated elliptical shape. There is no known closed form solution for this, although several approximations have been proposed for particular cases [2]. Here we use a numerical solution to the problem, along with measured values of losses, transmitter power, antenna radiation pattern and gain.

Results from the 5 October 2010 data are shown in Table 2. The differences in clutter-to-noise ratio (CNR) are due to the higher noise figure in the monostatic receiver caused by the Transmit/Receive $(\mathrm{T} / \mathrm{R})$ switch and the larger cell area in the bistatic configuration. The results in Table 2 show that there was no significant difference between monostatic and bistatic reflectivities with changing bistatic geometry, except at a bistatic angle of $90^{\circ}$. This lack of variation of backscatter coefficient with bistatic angle is not typical of bistatic clutter data in the literature, as described in [1]. However, there are some similar results reported in [8] for vertically polarised, low sea state returns.

Amplitude statistics: It is known that the probability density function (PDF) of the amplitude of monostatic sea clutter is generally longertailed than that of the Rayleigh distribution. Several distributions have been proposed to describe their PDF; the compound K-distribution and its variants $[4,9]$ have been widely used, and are used here. The data was fitted to the $\mathrm{K}+$ noise distribution, the PDF of which in terms of intensity can be written as:

$$
P_{C+N}(I)=\int_{0}^{\infty} \frac{b^{v} x^{\nu-1}}{\Gamma(\nu)} \frac{\exp (b x)}{x+p_{n}} \exp \left(\frac{-I}{x+p_{n}}\right) d x
$$

where $I$ is the intensity, $x$ the local clutter power, $v$ the shape parameter, $p_{n}$ the noise power, $b=v / \sigma_{B W}$ the scale parameter and $\sigma_{B W}$ is the average clutter power. The clutter to noise ratio is given by $C N R=\sigma_{B W} / p_{n}$

A Fibonacci search algorithm was used to find the best fit shape parameter, minimising the rms error between the CDF of the data and the numerically generated $\mathrm{K}+$ noise data [10]. Figs. 2 and 3 show the fits of the model to the measured data for the monostatic and bistatic data, respectively, for a bistatic angle of $30^{\circ}$. It can be seen that the fit, as measured by the rms error, is good in both cases, but better for the bistatic data. In all cases in Table 2 the value of the shape parameter for the bistatic clutter $\nu_{\mathrm{B}}$ is greater than that for monostatic clutter $v_{\mathrm{M}}$, showing that the bistatic clutter is less 'spiky'. This result is important, because it suggests that a bistatic geometry will allow a lower detection threshold for a given probability of false alarm, and hence give improved detection performance of weak targets against a clutter background compared to the conventional monostatic geometry. Similar results were obtained in analysing data from proving trials conducted in the UK at Peacehaven, Sussex, prior to deployment of the radar to South Africa, in different sea state conditions and grazing angles. 


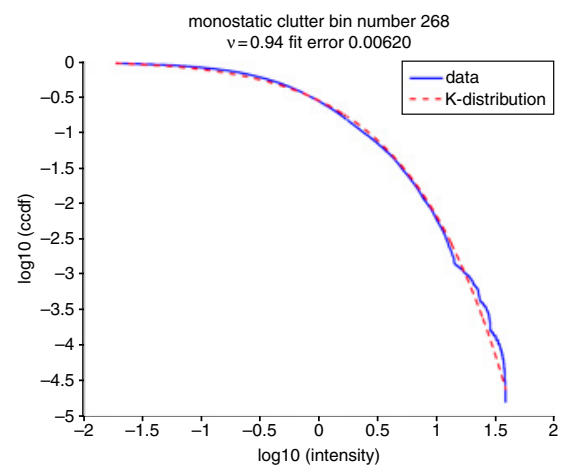

Fig. 2 Fit of $K+$ noise model to measured monostatic clutter Horizontal axis is log of intensity normalised to mean; vertical axis is $\log$ of complementary cumulative distribution function

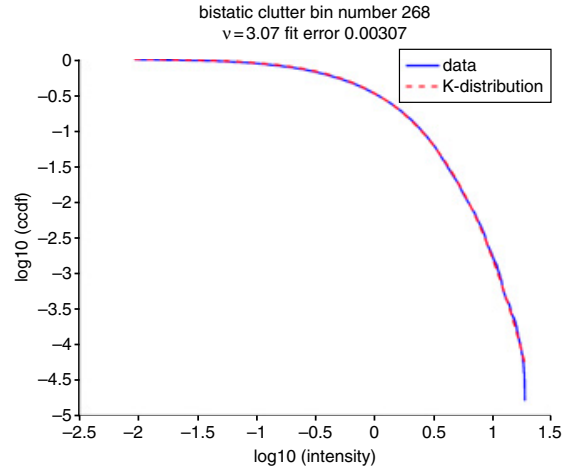

Fig. 3 Fit of $K+$ noise model to bistatic clutter measured simultaneously to that of Fig. 2

Horizontal axis is $\log$ of intensity normalised to mean; vertical axis is $\log$ of complementary cumulative distribution function

Conclusion: We present an analysis of simultaneously-recorded bistatic and monostatic $2.4 \mathrm{GHz}$ vertically-polarised sea clutter data. Fits of the $\mathrm{K}+$ noise distribution to the data show that in all cases the shape parameter of the distribution is significantly greater for the bistatic clutter than for monostatic. It remains to evaluate the improvement in detection performance due to this effect. This will have to take into account not only the variation in clutter parameters with bistatic geometry, but also those of the target. It is believed that this is the first published statistical analysis of simultaneous bistatic and monostatic sea clutter.

Acknowledgments: The authors acknowledge the support of the US Office of Naval Research (Global), Thales UK and Thales
Netherlands, and assistance of the South African National Defence Force (Navy), the Council for Scientific and Industrial Research (South Africa), the Institute for Maritime Technology (South Africa), the South African National Parks Board, and National Ports Authority (TNPA) for providing wind and wave data.

(C) The Institution of Engineering and Technology 2011

1 March 2011

doi: 10.1049/el.2011.0557

One or more of the Figures in this Letter are available in colour online.

W.A. Al-Ashwal, A. Balleri, H.D. Griffiths, W.J. Miceli, M. Ritchie and K. Woodbridge (Department of Electronic and Electrical Engineering, University College London, United Kingdom)

E-mail: w.al-ashwal@ee.ucl.ac.uk

C.J. Baker (Australian National University, Canberra, Australia)

R. Harmanny (Thales Nederland)

M. Inggs and J.S. Sandenbergh (Department of Electrical Engineering, University of Cape Town, South Africa)

A. Stove and S. Watts (Thales UK)

R.J.A. Tough and K.D. Ward (Igence Ltd, Malvern, United Kingdom)

\section{References}

1 Griffiths, H.D., Al-Ashwal, W., Ward, K.D., Tough, R.J.A., Baker, C.J. and Woodbridge, K.: 'Measurement and modelling of bistatic radar sea clutter', IET Radar Sonar Navig., 2010, 4, (2), pp. 280-292

2 Weiner, M.: 'Clutter', Chapter 9, in Willis, N.J., Griffiths, H.D. (Eds) 'Advances in bistatic radar' (SciTech Publishing, Raleigh, NC, 2007)

3 Ward, K.D., and Watts, S.: 'Use of sea clutter models in radar design and development', IET Radar Sonar Navig., 2010, 4, (2), pp. 146-157

4 Ward, K.D., Tough, R.J.A., and Watts, S.: 'Sea clutter: Scattering, the K-distribution and radar performance' (IET, 2006)

5 Derham, T.E., Doughty, S., Woodbridge, K., and Baker, C.J.: 'Realisation and evaluation of a low cost netted radar system'. Proc. CIE Int. Radar Conf., Shanghai, China, 2006, pp. 1-4

6 Doughty, S.R.: 'Development and performance evaluation of a multistatic radar system', Ph.D. dissertation, 2007, University College London

7 Sandenbergh, J.S., and Inggs, M.: 'A common view GPSDO to synchronize netted radar'. Proc IET Int. Conf. RADAR 2007, Edinburgh, UK, October 2007, pp. 1-5

8 Ewell, G., and Zehner, S.: 'Bistatic sea clutter return near grazing incidence'. Proc. IEE Int. Conf. RADAR 82, IEE Conf. Publ. No. 216, October 1982, pp. $188-192$

9 Watts, S.: 'Radar detection prediction for targets in both K-distributed sea clutter and thermal noise', IEEE Trans. Aerosp. Electron. Syst., 1987, AES-23, (2), pp. 40-45

10 Davidson, G.: 'Radar works', http://www.radarworks.com/, accessed January 2011 